to operating room personnel. Maintenance with isoflurane and after surgery risk of pain score of $5-7$ so the patient performed Transversus Abdominis Block of $0.2 \%$ ropivacaine and Clonidine $30 \mathrm{mcg}$ with $5 \mathrm{ml}$ for each side. Transversus abdominis plane block is a regional technique, ultrasound guidance has increased the safety and efficacy of blocks. Ultrasonography allows real-time visualization of anatomical structures, guides the blocking procedure, and shows the spread of the local anaesthetic solution. A more rapid onset of block using less local anaesthetic solution is particularly attractive for paediatrics where most blocks are sited in anaesthetized patients.

Conclusions Intraoperative management including induction, intubation, and extubation should be considered to reduce viral exposure. Postoperative management includes optimal hemodynamic and adequate analgesic monitoring. Regional techniques provide excellent postoperative analgesia in pediatric.

\section{REGIONAL METHODS OF PAIN RELIEF IN CHILDREN WITH CEREBRAL PALSY IN HIP SURGERIES}

${ }^{1} V$ Evreinov*, ${ }^{2}$ T Zhirova. ${ }^{1}$ Federal State Budgetary Institution 'National Ilizarov Medical Research Centre for Traumatology and Ortopaedics' Ministry Healthcare, Kurgan, Russian Federation; ${ }^{2}$ State-funded Healthcare Institution of Sverdlovsk region 'Center for specialized types of medical care 'Ural Institute of Traumatology and orthopedics named after V. D. Chaklin, Yekaterinburg, Russian Federation

10.1136/rapm-2021-ESRA.227

Background and Aims Prolonged epidural analgesia (PEA) is effective in surgeries done for spastic hip dislocation in children with cerebral palsy and severe forms of motor

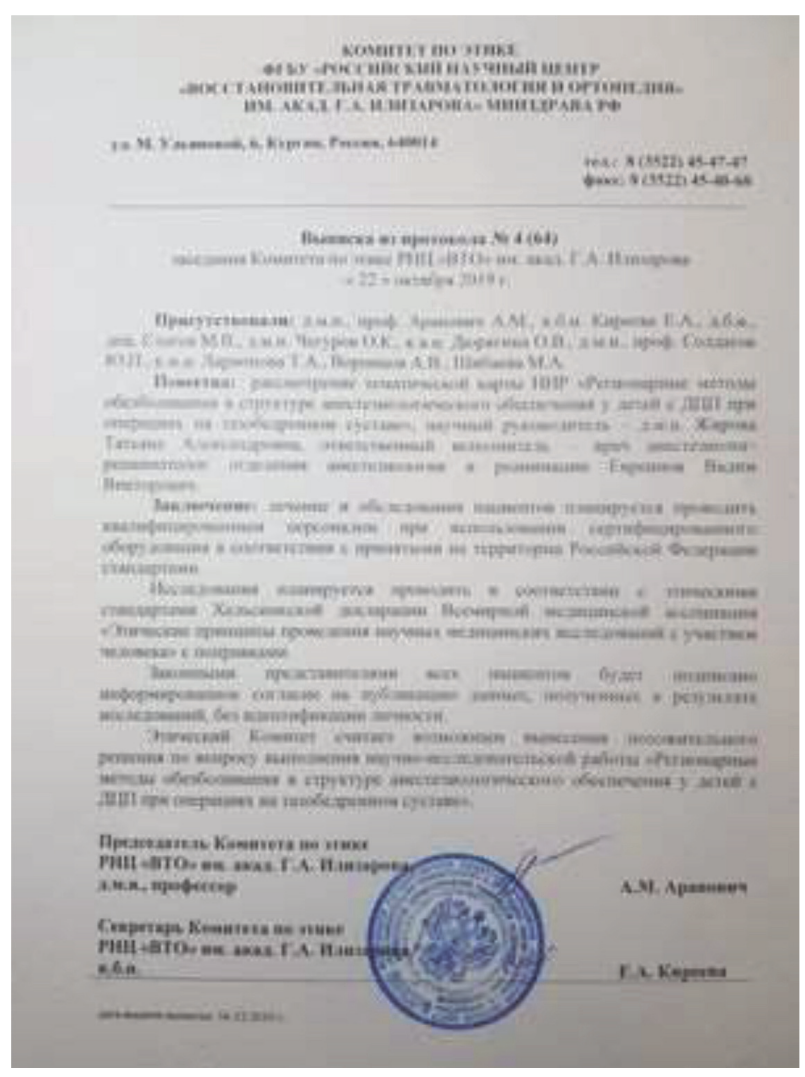

Abstract 227 Figure 1 dysfunction: IV-V levels according to Gross Motor Function Classification System (GMFSC). Sometimes PEA becomes impossible due to anatomic peculiarities and contraindications. The purpose of this study is to compare different methods of regional analgesia and sedation in children with cerebral palsy for hip surgeries combined with muscle-tendon plasty.

Methods One hundred and one children were included into prospective randomized study, which was approved by local Ethics Committee. Patients received general anesthesia supplemented: PEA and analgo-sedation with fentanyl (group EAA, 32 patients); PEA and sedation with benzodiazepines (group EABS, 37 patients); prolonged femoral nerve block with single ischial nerve blockade (group PCABS, 32 patients). Intensity of pain according to r-FLACC scale, degree of agitation and sedation according to RASS scale, depth of sedation according to Ramsay were evaluated after waking-up, in 6 hours after surgery and on 1 POD. We calculated prescription of NSAIDs and opioids. ANOVA, $\square^{2}$ were used.

Results Paracetamol was prescribed to 15 patients (47\%) in group EAA, $32(86.5 \%)$ in group EABS, 31 (97\%) in group PCABS $(p<0.001)$. Patients EABS and PCABS did not need opioids. There were no differences between groups according to scales r-FLACC, RASS and Ramsay ( $p>0.05)$.

Conclusions PEA, prolonged femoral nerve analgesia together with single ischial nerve blockade in combination with NSAIDs are equally effective in hip surgeries in children with cerebral palsy. Sedation with benzodiazepines decreases the need for narcotic analgesics.

\section{OPIOID SPARING ANAESTHESIA AND ANALGESIA TECHNIQUES; A QUALITY IMPROVEMENT PROJECT}

D Liotiri* ${ }^{*}$ N Attaway, S Soobhug, W Caddye. Brighton and Sussex University Hospitals NHS Trust, Brighton, UK

\subsection{6/rapm-2021-ESRA.228}

Background and Aims Overreliance on opioids to treat postoperative pain and increased availability of opioids in the community have contributed to the opioid epidemic (1). For many patients, the source of initial exposure to opioids is the perioperative period. The aim of this project was to identify local practice and introduce ways to minimise opioids during anaesthesia and recovery.

Methods We conducted a two-week snapshot audit in November 2019 at Brighton and Sussex University Hospitals (BSUH) (UK). The audit was registered with BSUH anaesthetic department Audit and Quality Improvement. Ethics approval was not required. We collected data on opioid use in four theatres and recovery. We also conducted a literature search to identify best available evidence on non-opioid adjuncts.

Results Median opioid use in each theatre and recovery are presented in table 1 . In recovery, 1 in 3 patients required more than $10 \mathrm{mg}$ intravenous morphine equivalents (IVME), and more than 1 in 10 patients received more than $20 \mathrm{mg}$ IVME. We compiled evidence-based opioid sparing anaesthesia and analgesia guides for use in theatres and recovery (Figure $1)$.

Conclusions Although there are no standards to compare with, we believe that these results indicate high opioid use during anaesthesia and recovery. The risk of opioid dependence and side effects that may adversely affect surgical outcomes should be taken into account before prescribing opioids. There is a 

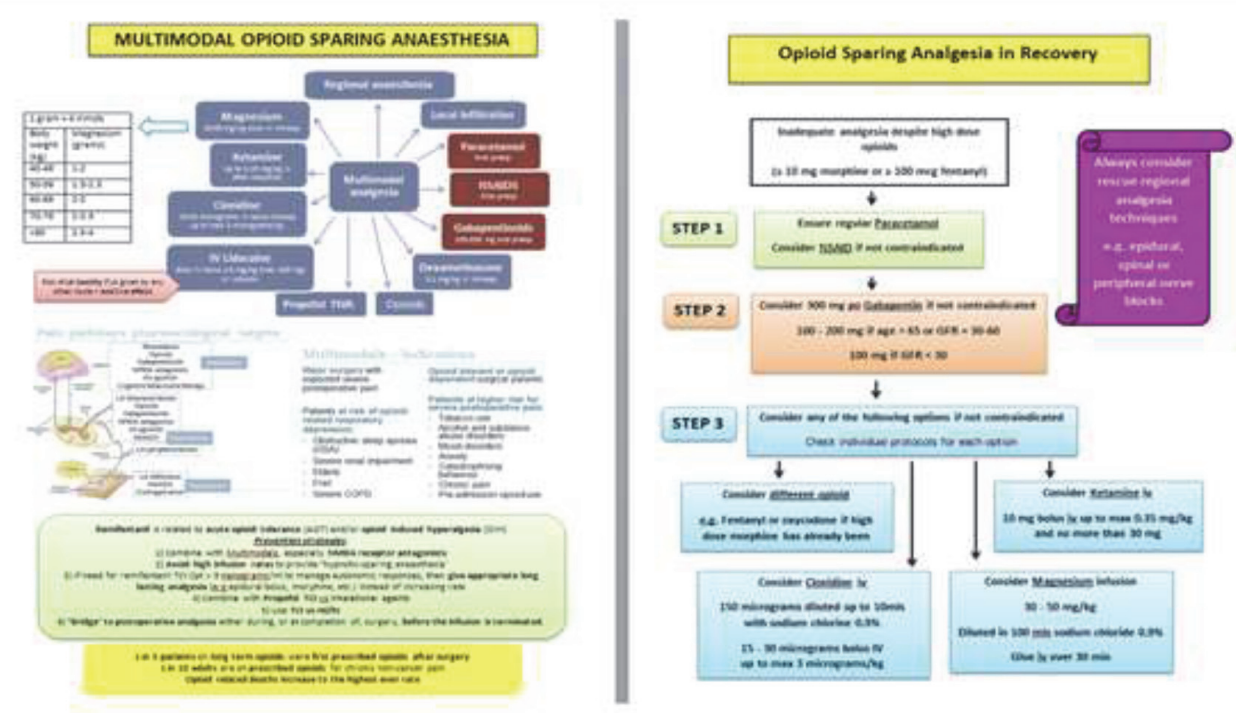

Abstract 228 Figure 1

Abstract 228 Table 1 Opioid use in theatres and recovery during the first two weeks of November 2019. IVME=IV morphine equivalents, $n=$ number of patients, median=median opioid use, PACU=post anaesthesia care unit

\begin{tabular}{|c|c|c|c|}
\hline BSUH AREA & $\begin{array}{c}\text { IVME } \\
\text { (mg) }\end{array}$ & $\mathbf{n}$ & Median (min-max) (mg) \\
\hline $\begin{array}{c}\text { Theatre 1 } \\
\text { (orthopaedic trauma) }\end{array}$ & 555.5 & 30 & $20(2.5-38)$ \\
\hline $\begin{array}{c}\text { Theatre 2 } \\
\text { (complex ENT) }\end{array}$ & 225.5 & 16 & $10(5-30)$ \\
\hline $\begin{array}{c}\text { Theatre 4 } \\
\text { (Emergencies) }\end{array}$ & 806.5 & 43 & $20(5-40)$ \\
\hline $\begin{array}{c}\text { Theatre 5 } \\
\text { (Major gynaccology and gencral } \\
\text { oncology surgery) }\end{array}$ & 260.5 & 16 & $12.5(5-30)$ \\
\hline PACU & 870 & 85 & $\mathbf{8 ( 2 - 4 5 . 4 )}$ \\
\hline
\end{tabular}

plethora of non-opioid adjuncts which may facilitate opioidfree or opioid-light anaesthesia and analgesia. Further work is required to investigate if anaesthesia and recovery opioid sparing protocols can improve patient outcomes.

\section{INSTITUTIONAL ENHANCED RECOVERY PROGRAM QUALITY PAIN AUDIT}

P-J Windal*, S Coppens. UZ Leuven, Leuven, Belgium

10.1136/rapm-2021-ESRA.229

Background and Aims Pain remains a major complaint in the immediate post-operative phase. Despite enhanced recovery protocols (ERP) and procedure specific pain therapy up to $80 \%$ of patients experience significant uncontrolled pain. Aim of this quality control pain audit was assessing pain intensity in institutional developed multimodal perioperative care pathways. Analyses of efficacy locoregional analgesia, management and protocol adherence. Determine possible improvements of guidelines and procedures

Methods Prospective data were collected on 118 patients undergoing six types of surgery. Pain scores were evaluated on

postoperative care unit (PACU) and first five days following surgery. Primary outcome was worst recorded pain score. Secondary outcomes included median pain on ward, incidence of postoperative nausea and vomiting, time to first mobilization. Following procedures were included: minimal invasive cardiac surgery, video assisted thoracic surgery, esophageal resection, total knee arthroplasty, laparoscopic colorectal surgery and robotic prostatectomy.

Results Numeric Rating Scale higher then three was observed in $89 \%$ of study population. At PACU esophageal resection showed worst pain scores despite neuraxial analgesia (Median: 7, IQR 5-8). Total knee arthoplasty resulted in worst pain scores on ward following surgery (median: 7, IQR 5-8). Disappointingly only $25 \%$ of patients received pain medication following institutional ERP guidelines. Current ERP-guideline are similar to ERAS guidelines found in literature.

Conclusions Multimodal pain therapy should become standard of care. Constant evaluation of pain guidelines are necessary. Audits and evaluation of protocol adherence next to clear guidelines and managing patient information, expectations are pivotal.

\section{CLONIDINE AS AN ADJUNCT TO LEVOBUPIVACAINE FOR PUDENDAL BLOCK DURING PROCTOLOGICAL SURGERY: A PROSPECTIVE, RANDOMISED, PILOT STUDY}

M Gambassi*, M Suball, M Bortes, P Kapessidou. CHU Saint Pierre, Brussels, Belgium

10.1136/rapm-2021-ESRA.230

Background and Aims Proctological surgery is associated with high-score postoperative pain (VAS $>5$ ) [1].

Pudendal block reduces pain and allows a faster return to normal activity [2].

Clonidine has been suggested to improve the quality and duration of peripheral blocks [3 4 ].

This study investigates whether adding clonidine to levobupivacaine in proctological surgery reduces postoperative consumption of analgesics.

Methods After local ethics committee approval and signed informed consent, adults ASA I/II, scheduled for proctologic 